\title{
Equations defining reducible Kummer surfaces in $\mathbb{P}^{5}$
}

\author{
by Tomasz Szemberg (Kraków and Erlangen)
}

\begin{abstract}
Principally polarized abelian surfaces are the Jacobians of smooth genus 2 curves or of stable genus 2 curves of special type. In [S] we studied equations describing Kummer surfaces in the case of an irreducible principal polarization on the abelian surface. The aim of this note is to give a treatment of the second case. We describe intermediate Kummer surfaces coming from abelian surfaces carrying a product principal polarization. In Proposition 12 we give explicit equations of these surfaces in $\mathbb{P}^{5}$.
\end{abstract}

1. Introduction. This note is a continuation of [S1]. Here we study equations of Kummer surfaces induced by some partial linear system arising from a reducible principal polarization on an abelian surface. With a slight abuse of language we call the resulting surfaces reducible intermediate Kummer surfaces. These surfaces are projections of singular abelian surfaces which are complete intersections of 4 quadrics in $\mathbb{P}^{6}$ described first by Adler and van Moerbeke in [AvM1] and [AvM2]. The abelian surfaces were studied extensively from the algebro-geometric point of view by Barth in $[\mathrm{B}]$.

For preliminaries we refer to $[\mathrm{M}]$ and [S1]. As far as possible we stick to the notation of our previous paper. We recall it briefly in the next section.

The base field throughout the note is the field $\mathbb{C}$ of complex numbers.

2. The set-up. In [S1] we studied equations of Kummer surfaces coming from the Jacobians of smooth genus two curves. Let now $A$ be the product of elliptic curves $F_{1}$ and $F_{2}$ and $\Theta=F_{1}+F_{2}$ be a symmetric divisor on $A$ with $\mathcal{L}=\mathcal{O}_{A}(\Theta)$. Thus $(A, \mathcal{L})$ is a principally polarized abelian surface. Let us denote the halfperiods on $A$ as shown in Figure 1.

1991 Mathematics Subject Classification: 14J28, 14J70.

Key words and phrases: Kummer surfaces, intersections of quadrics.

Supported by Daimler-Benz Stiftung project number 2.92.34 and KBN grant number 210779101. 


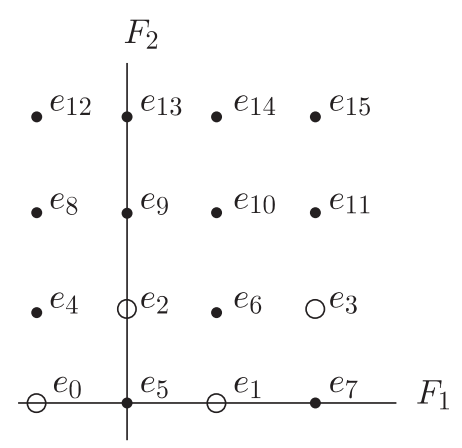

Fig. 1

Here $e_{0}$ is a neutral element of a torus action of $A$ on itself and in this convention $e_{3}=e_{1}+e_{2}$. We denote by $G$ the subgroup of the halfperiods on $A$ consisting of $e_{0}, \ldots, e_{3}$. This subgroup can be lifted in a natural way to the total space of $\mathcal{O}_{A}(4 \Theta)$. Thus $G$ acts on $H^{0}\left(\mathcal{O}_{A}(4 \Theta)\right)$. The liftings of $e_{1}$ and $e_{2}$ can be chosen to be again involutions, which we denote by $\sigma$ and $\tau$ respectively.

Let $B l: \widetilde{A} \rightarrow A$ be the blowing up of $A$ at $e_{0}, \ldots, e_{3}$ and $B l_{s}: A_{s} \rightarrow A$ the blowing up at all 16 halfperiods. In both cases we denote the exceptional divisor over $e_{i}$ by $E_{i}$. Let $\widetilde{\iota}=B l^{*}(\iota)$ and $\iota_{s}=B l_{s}^{*}(\iota)$, where $\iota: A \ni a \rightarrow$ $-a \in A$ is the inverse element mapping on $A$. The quotients $\widetilde{K}=\widetilde{A} / \widetilde{\iota}$ and $K_{s}=A_{s} / \iota_{s}$ are called the intermediate and the smooth Kummer surface of $A$ respectively. The quotient mappings are denoted by $\widetilde{\pi}$ in the first case and by $\pi_{s}$ in the second.

In what follows we deal mostly with the surface $\widetilde{K}$ which is singular. If it appears to be disturbing one can always think of divisors and line bundles on $\widetilde{K}$ as push-downs from the smooth model $K_{s}$. This should exclude any possible confusion.

For a symmetric divisor $D$ on an abelian surface $A$ we denote by $H^{0}(D)^{\text {ev }}$ and $H^{0}(D)^{\text {odd }}$ the eigenspaces of 1 and -1 respectively of the mapping $H^{0}(D) \ni s \rightarrow \iota_{L} s \iota \in H^{0}(D)$. Here $\iota_{L}$ is the lifting of $\iota$ to an involution on the total space of $L=\mathcal{O}_{A}(D)$. The elements of $H^{0}(D)^{\mathrm{ev}}$ are called even sections, and elements of $H^{0}(D)^{\text {odd }}$ odd sections of the line bundle $L$.

For a divisor $D$ on a surface $X$, a point $x \in X$ and a natural number $n$ we denote by $|D-n x|$ those divisors in the linear system $|D|$ which pass through $x$ with multiplicity at least $n$. Equivalently one can think of sections in $\mathcal{O}_{X}(D)$ vanishing at $x$ to order at least $n$ or of sections in the sheaf $\mathcal{I}_{x}^{\otimes n} \cdot \mathcal{O}_{X}(D)$, where $\mathcal{I}_{x}$ is the ideal sheaf of $x$.

3. The linear systems on $A$ and $\widetilde{K}$. We are interested in the equations of the image $X$ of $\widetilde{K}$ in $\mathbb{P}^{5}$ under the morphism $\varphi: \widetilde{A} \rightarrow \mathbb{P}^{5}$ defined by the linear system $L=\left|4 B l^{*} \Theta-2\left(E_{0}+E_{1}+E_{2}+E_{3}\right)\right|^{\text {ev }}$. This morphism factors 
over $\psi: \widetilde{K} \rightarrow \mathbb{P}^{5}$. Moreover, both mappings are $G$-equivariant. We begin the study of the linear system $L$ with the following

Proposition 1. For $L=\left|4 B l^{*} \Theta-2\left(E_{0}+E_{1}+E_{2}+E_{3}\right)\right|^{\text {ev }}$ we have $h^{0}(L)=6$.

Proof. $H^{0}(4 \Theta)$ can be written as a direct sum $H^{0}(4 \Theta)^{\mathrm{ev}} \oplus H^{0}(4 \Theta)^{\text {odd }}$. According to $\left[\mathrm{LB}\right.$, formula 4.7.5] we have $h^{0}(4 \Theta)^{\mathrm{ev}}=10$ and $h^{0}(4 \Theta)^{\text {odd }}=6$. The linear system $\left|4 \Theta-\left(e_{0}+\ldots+e_{3}\right)\right|$ has dimension 12 since the four imposed conditions are clearly independent. Moreover, we also have

$\left|4 \Theta-\left(e_{0}+\ldots+e_{3}\right)\right|=\left|4 \Theta-\left(e_{0}+\ldots+e_{3}\right)\right|^{\text {ev }} \oplus\left|4 \Theta-\left(e_{0}+\ldots+e_{3}\right)\right|^{\text {odd }}$.

Since $4 \Theta$ is totally symmetric the odd sections vanish at each halfperiod to order at least one. Hence $H^{0}(4 \Theta)^{\text {odd }}=\left|4 \Theta-\left(e_{0}+\ldots+e_{3}\right)\right|^{\text {odd }}$ and it follows that $\operatorname{dim}\left|4 \Theta-\left(e_{0}+\ldots+e_{3}\right)\right|^{\mathrm{ev}}=12-6=6$. This proves the assertion since again by the total symmetry $\left|4 \Theta-\left(e_{0}+\ldots+e_{3}\right)\right|^{\mathrm{ev}}=\left|4 \Theta-2\left(e_{0}+\ldots+e_{3}\right)\right|^{\mathrm{ev}}$ and the system in question is the pull-back under the blowing-up of the last system.

The following lemma turns out to be useful in the explicit computation of the action of $G$ on $L$.

Lemma 2. Let $\sigma^{ \pm}, \tau^{ \pm}$be the eigenspaces of \pm 1 for $\sigma, \tau$ respectively. Then $\operatorname{dim} \sigma^{+}=\operatorname{dim} \tau^{+}=4$ and $\operatorname{dim} \sigma^{-}=\operatorname{dim} \tau^{-}=2$.

Pr o of. Since the procedure for $\sigma, \tau$ is the same we consider $\sigma$ only.

Let $w_{1}, w_{2}$ be complex numbers with $\operatorname{Im} w_{i}>0$ and $F_{i}=\mathbb{C} /\left(\mathbb{Z} w_{i} \oplus \mathbb{Z}\right)$ for $i=1,2$. Then $A=\mathbb{C}^{2} / \Lambda$, where

$$
\Lambda=\left(\begin{array}{c}
w_{1} \\
0
\end{array}\right) \mathbb{Z} \oplus\left(\begin{array}{l}
1 \\
0
\end{array}\right) \mathbb{Z} \oplus\left(\begin{array}{c}
0 \\
w_{2}
\end{array}\right) \mathbb{Z} \oplus\left(\begin{array}{l}
0 \\
1
\end{array}\right) \mathbb{Z}
$$

is a principally polarized abelian surface and $A=F_{1} \times F_{2}$. We denote the period matrix of $A$ by $\Pi$.

Consider the matrices

$$
\Pi_{\varepsilon}=\left(\begin{array}{cccc}
w_{1} & 1 & \varepsilon & 0 \\
\varepsilon & 0 & w_{2} & 1
\end{array}\right) .
$$

For $\varepsilon \in D=\left\{z \in \mathbb{C}:|z|<\left(\operatorname{Im} w_{1} \operatorname{Im} w_{2}\right)^{1 / 2}\right\}$ the matrix $\Pi_{\varepsilon}$ defines a principally polarized abelian surface $A_{\varepsilon}$ (see [LB, 4.2]). For $\varepsilon \neq 0$ the surface $A_{\varepsilon}$ is not a product of elliptic curves (compare [LB, 10.6.1]). Hence it must be the Jacobian surface of some smooth curve $C_{\varepsilon}$ of genus 2 . We denote by $\Theta_{\varepsilon}$ the image of $C_{\varepsilon}$ in $A_{\varepsilon}$ under the Abel-Jacobi mapping. Thus we have a family $\mathcal{A}=\bigcup A_{\varepsilon}$ of principally polarized abelian surfaces $\left(A_{\varepsilon}, \Theta_{\varepsilon}\right)$ over the disc $D$ in the complex plane. Let $\pi: \mathcal{A} \rightarrow D$ be the obvious mapping $A_{\varepsilon} \ni x \rightarrow \varepsilon \in D$.

There is a section $s_{0}: D \rightarrow \mathcal{A}$ such that $s_{0}(\varepsilon)=$ a neutral element $e_{0}^{\varepsilon}$ of $A_{\varepsilon}$. This section can be translated to the sections $s_{1}, s_{2}$ in such 
a way that for $i=1,2$ we have $s_{i}(\varepsilon)=e_{i}^{\varepsilon}$, where $e_{i}^{\varepsilon}$ are two even halfperiods on $A_{\varepsilon}$ and $e_{i}^{0}=e_{i}$. Thus for each $\varepsilon$ we also have the involutions $\sigma_{\varepsilon}, \tau_{\varepsilon}$ operating on $L_{\varepsilon}=H^{0}\left(\mathcal{I}_{\varepsilon} \cdot \mathcal{O}_{A_{\varepsilon}}\left(4 \Theta_{\varepsilon}\right)\right)^{\text {ev }}$, where $\mathcal{I}_{\varepsilon}$ denotes the ideal sheaf of $e_{0}^{\varepsilon}, e_{1}^{\varepsilon}, e_{2}^{\varepsilon}, e_{3}^{\varepsilon}=e_{1}^{\varepsilon}+e_{2}^{\varepsilon}$. By Proposition 1 and [S1, Proposition 6] the vector spaces $L_{\varepsilon}$ have dimension 6 for each $\varepsilon \in D$. These vector spaces patched together yield a vector bundle $\mathcal{L}$ on $\mathcal{A}$. It can be easily seen that the mapping $\widetilde{\sigma}(x):=\sigma_{\pi(x)}(x)$ for $x$ in the total space of $\mathcal{L}$ is a vector bundle automorphism. Moreover, $\widetilde{\sigma}$ is an involution. Let $\mathcal{E}_{\lambda}$ denote $\operatorname{ker}\left(\widetilde{\sigma}-\lambda \operatorname{id}_{\mathcal{L}}\right)$ for $\lambda= \pm 1$. Then according to Grauert's semicontinuity theorem [BPV, Theorem 1.8.5.ii], $\operatorname{dim} \mathcal{E}_{ \pm 1}(\varepsilon)=\operatorname{dim} \sigma_{\varepsilon}^{ \pm 1}$ are upper semicontinuous functions of $\varepsilon$, hence these dimensions cannot drop. But they cannot jump up either because $1,-1$ are the only eigenvalues of $\widetilde{\sigma}$ and $\operatorname{dim} \sigma_{\varepsilon}^{+1}+\operatorname{dim} \sigma_{\varepsilon}^{-1}=H^{0}\left(L_{\varepsilon}\right)=H^{0}\left(\pi_{*} \mathcal{L}\right)$ according to the base change theorem [BPV, Theorem 1.8.5.iv]. The assertion follows now from [S1, Prop. 6].

Lemma 3. Let $M=\left|4 \Theta-2\left(e_{0}+\ldots+e_{15}\right)\right|$. Then $h^{0}(M)=1$.

Proof. The divisor $D=\Theta+t_{e_{3}}^{*} \Theta+t_{e_{9}}^{*} \Theta+t_{e_{14}}^{*} \Theta$ is clearly in $M$, hence $h^{0}(M) \geq 1$. Let $x \in A \backslash \operatorname{supp} D$. If $h^{0}(M) \geq 2$ then there is an effective divisor $D^{\prime} \in M$ such that $x \in \operatorname{supp} D^{\prime}$. It follows that $D \neq D^{\prime}$. On the other hand, D. $D^{\prime}=4 \Theta .4 \Theta=32$ and $D \cap D^{\prime}$ contains all 16 halfperiods with multiplicity at least 4 . Hence the two divisors must have common components. A somehow tedious computation on the components of $D, D^{\prime}$ shows that $D=D^{\prime}$. Hence $h^{0}(M)=1$.

Let $s_{0}$ be a generator of $H^{0}(\Theta)$. Since the line bundles $\mathcal{O}_{A}(2 \Theta)$ and $\mathcal{O}_{A}\left(2 t_{e_{\bullet}}^{*} \Theta\right)$ are isomorphic for any halfperiod $e_{\bullet}$ the section $s_{0}^{2}$ can be translated to a section $s_{\bullet}^{2}$ doubly vanishing on $\Theta_{\bullet}=t_{e}^{*}(\Theta)$. These translates are canonically defined as soon as the theta structure is fixed. Furthermore, let $w_{4}=B l^{*} s$ for some section $s$ with the divisor of $s$ in the linear system $M$. Let us note that $w_{4}$ is thus fixed up to a constant in view of the previous lemma.

Now we are in a position to write a basis for the linear system $L$ explicitly.

Proposition 4. The sections $w_{1}=s_{0}^{2} s_{1}^{2}, w_{2}=s_{2}^{2} s_{3}^{2}, w_{3}=s_{1}^{2} s_{2}^{2}+s_{0}^{2} s_{3}^{2}$, $w_{4}, w_{5}=s_{0}^{2} s_{2}^{2}$ and $w_{6}=s_{1}^{2} s_{3}^{2}$ form a basis of $H^{0}(L)$ in which $\sigma$ and $\tau$ are represented by the matrices

$$
\sigma=\left[\begin{array}{cccccc}
1 & 0 & & & & \\
0 & 1 & & & & \\
& & 1 & 0 & & \\
& & 0 & -1 & & \\
& & & & 0 & 1 \\
& & & & 1 & 0
\end{array}\right], \quad \tau=\left[\begin{array}{cccccc}
0 & -1 & & & & \\
-1 & 0 & & & & \\
& & -1 & 0 & & \\
& & 0 & 1 & & \\
& & & & 1 & 0 \\
& & & & 0 & 1
\end{array}\right] .
$$


Pr o of. It is convenient to view the zero sets of the above sections as in Figure 2.
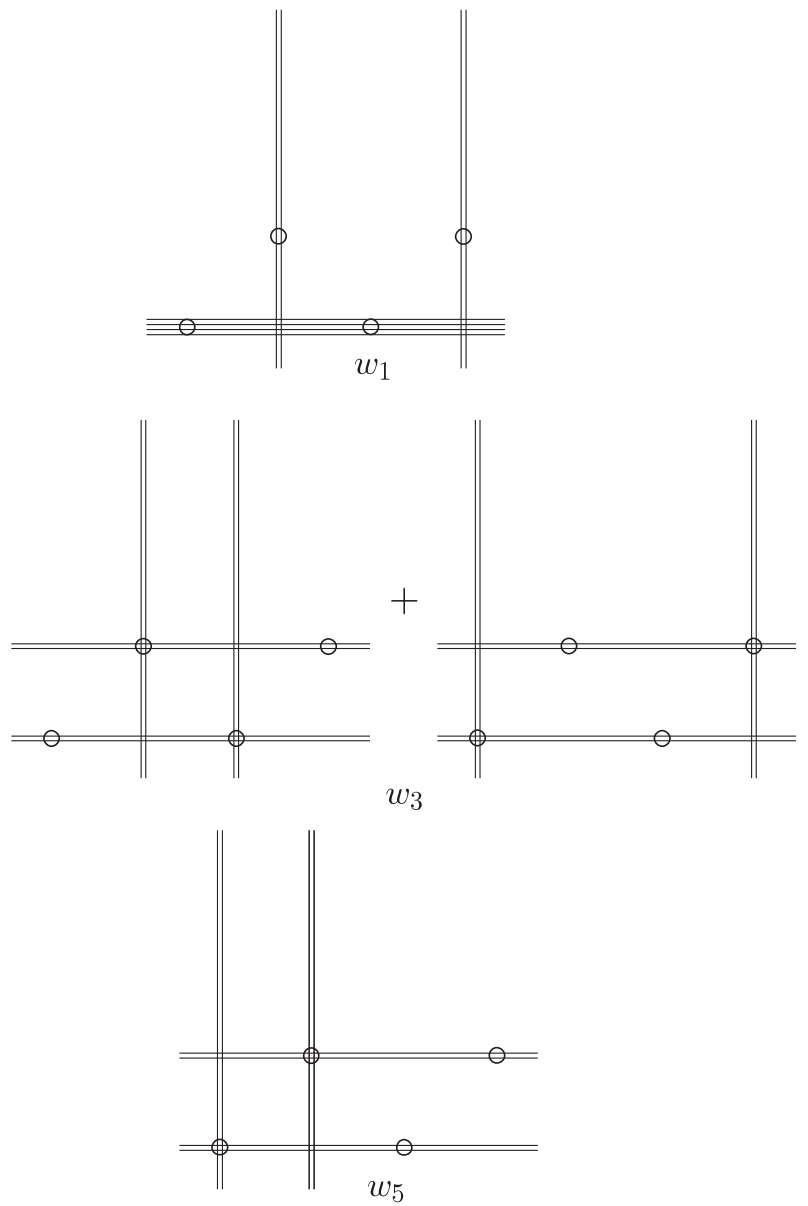
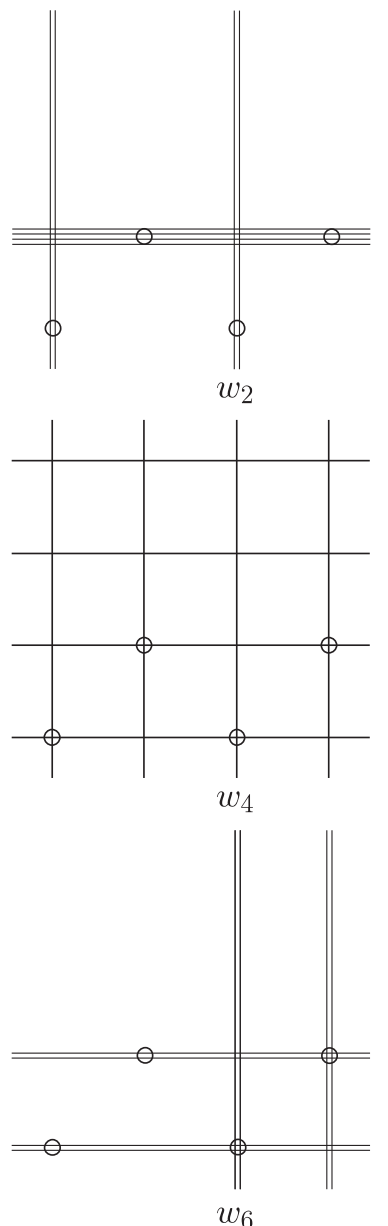

Fig. 2

It is clear that the sections $w_{1}, \ldots, w_{6}$ are in $L$. We prove the linear independence and the representation of $\sigma, \tau$ simultaneously.

Assume that

$$
\sum_{i=1}^{6} \lambda_{i} w_{i} \equiv 0 .
$$

Restricting this identity to the elliptic curves $F_{1}$ and $t_{e_{2}}^{*}\left(F_{1}\right)$ we get at once $\lambda_{1}=\lambda_{2}=0$. Let $V=\operatorname{span}\left\{w_{3}, \ldots, w_{6}\right\}$. We are done if we show $\operatorname{dim} V=4$. Since $w_{3}, w_{5}, w_{6}$ are obviously linearly independent the contrary assumption is $\operatorname{dim} V=3$. Before we show that this is not possible we have to compute 
the action of $\sigma$ and $\tau$. The method of [S1, Prop. 6] applies directly to all sections but $w_{4}$ and we have:

\begin{tabular}{|r|r|r|r|r|r|}
\hline & $w_{1}$ & $w_{2}$ & $w_{3}$ & $w_{5}$ & $w_{6}$ \\
\hline$\sigma$ & $w_{1}$ & $w_{2}$ & $w_{3}$ & $w_{6}$ & $w_{5}$ \\
\hline$\tau$ & $-w_{2}$ & $-w_{1}$ & $-w_{3}$ & $w_{5}$ & $w_{6}$ \\
\hline
\end{tabular}

Now suppose $\operatorname{dim} V=3$. Then $V=V^{1} \oplus V^{-1}$, where $V^{ \pm 1}$ is the eigenspace of \pm 1 for $\tau$. What is more, $V^{1}=\operatorname{span}\left\{w_{5}, w_{6}\right\}, V^{-1}=\operatorname{span}\left\{w_{3}\right\}$. Since $w_{4}$ is invariant under $\tau$ up to sign we must have $w_{4} \in V^{1}$ or $w_{4} \in V^{-1}$. In the first case we would have $w_{4}=\alpha w_{5}+\beta w_{6}$. This is not possible: just compute its restriction to $F_{2}$ and $t_{e_{1}}^{*}\left(F_{2}\right)$. The second case is absurd, hence $\operatorname{dim} V=4$.

We must still compute the action of $\sigma$ and $\tau$ on $w_{4}$. As already mentioned, the only problem is to decide whether $w_{4}$ is a +1 or -1 eigenvector. According to Lemma 2 we must have $\sigma\left(w_{4}\right)=-w_{4}$ and $\tau\left(w_{4}\right)=w_{4}$.

The line bundle $\mathcal{O}_{A}\left(4 B l^{*} \Theta-2\left(E_{0}+\ldots+E_{3}\right)\right)$ defines a rank 2 vector bundle $\mathcal{M}=\widetilde{\pi}_{*} L$ on the Kummer surface $\widetilde{K}$ which splits into a direct sum of two line bundles $\mathcal{M}=\mathcal{M}^{+} \oplus \mathcal{M}^{-}$. There is a canonical isomorphism between $H^{0}(L)$ and $H^{0}\left(\mathcal{M}^{+}\right)$. Therefore we can denote the coordinates in the second space again by $w_{1}, \ldots, w_{6}$.

The next theorem describes the morphism defined by $\mathcal{M}^{+}$.

Proposition 5. The line bundle $\mathcal{M}^{+}$defines a birational morphism $\psi: \widetilde{K} \rightarrow X \subset \mathbb{P}^{5}$ which is an isomorphism away of the contracted curves $\widetilde{\pi}_{*} F_{1}$ and $\widetilde{\pi}_{*}\left(t_{e_{2}}^{*} F_{1}\right)$.

Pro of. The proof is based on Saint-Donat's theorem [S-D] and is similar to that of [S1, Prop. 8]. The only difference is the contraction of the two curves. To conclude, it is enough to observe that $\widetilde{\pi}_{*} F_{1} \cdot \mathcal{M}^{+}=\widetilde{\pi}_{*}\left(t_{e_{2}}^{*} F_{1}\right) \cdot \mathcal{M}^{+}$ $=0$. The projective coordinates of the image points can be easily computed. In the basis $w_{1}, \ldots, w_{6}$ they are

$$
\begin{aligned}
& p_{01}=\psi\left(\widetilde{\pi}_{*} F_{1}\right)=(0: 1: 0: 0: 0: 0), \\
& p_{23}=\psi\left(\widetilde{\pi}_{*}\left(t_{e_{2}}^{*} F_{1}\right)\right)=(1: 0: 0: 0: 0: 0) .
\end{aligned}
$$

4. Geometric properties. As in the case of an irreducible principal polarization of $A$ the surface $X$ contains 4 conics and 4 lines. They are now arranged in what we can call a degenerate $4_{3}$ configuration.

Lemma 6. The curves $C_{i}=\varphi\left(E_{i}\right)$ are smooth conics in $\mathbb{P}^{5}$ for $i=$ $0, \ldots, 3$.

Proof. For $i=0, \ldots, 3$ we have

$$
\operatorname{deg}\left(C_{i}\right)=\operatorname{deg}\left(\varphi\left(E_{i}\right)\right)=\mathcal{M}^{+} . D_{i}=2
$$


and $C_{i}$ is irreducible. It cannot be a double line according again to SaintDonat's theorem. Hence it is a smooth conic.

Lemma 7. The elliptic curves $t_{e_{i}}^{*}\left(F_{2}\right)$ go $2: 1$ under $\varphi$ onto lines $L_{3-i}$ for $i=0, \ldots, 3$. There are exactly 3 singular points of $X$ on each of these lines.

P r o of. It is enough to prove the lemma for a chosen curve, say $F_{2}$, since all the others are images of $\varphi\left(F_{2}\right)$ under the group $G$. Let $C=\widetilde{\pi}_{*} B l^{*} F_{2}$. Then we have

$$
\begin{aligned}
\operatorname{deg} L_{0} & =\mathcal{M}^{+} . C=\frac{1}{2}\left(4 B l^{*} \Theta-2\left(E_{0}+\ldots+E_{3}\right)\right) \cdot \widetilde{\pi}^{*} C \\
& =2 B l^{*}\left(F_{1}+F_{2}\right) \cdot\left(B l^{*} F_{2}-E_{2}\right)-1=1 .
\end{aligned}
$$

The two double points on $L_{0}$ are $\varphi\left(e_{9}\right)$ and $\varphi\left(e_{13}\right)$. The remaining singular point $\varphi\left(B l^{*}\left(t_{e_{2}}^{*}\left(F_{1}\right)\right)-E_{3}\right)$ is of type $A_{3}$.

COrollary 8. $\widetilde{X}$ has 8 double points of type $A_{1}$ and 2 singularities of type $A_{3}$.

In the sequel the geometric interpretation in Figure 3 of the degenerate $4_{3}$ configuration of lines and conics will be useful. The dotted lines are the conics, $\bullet$ denotes the $A_{1}$ singularities and $p_{01}, p_{23}$ are the $A_{3}$ singularities.

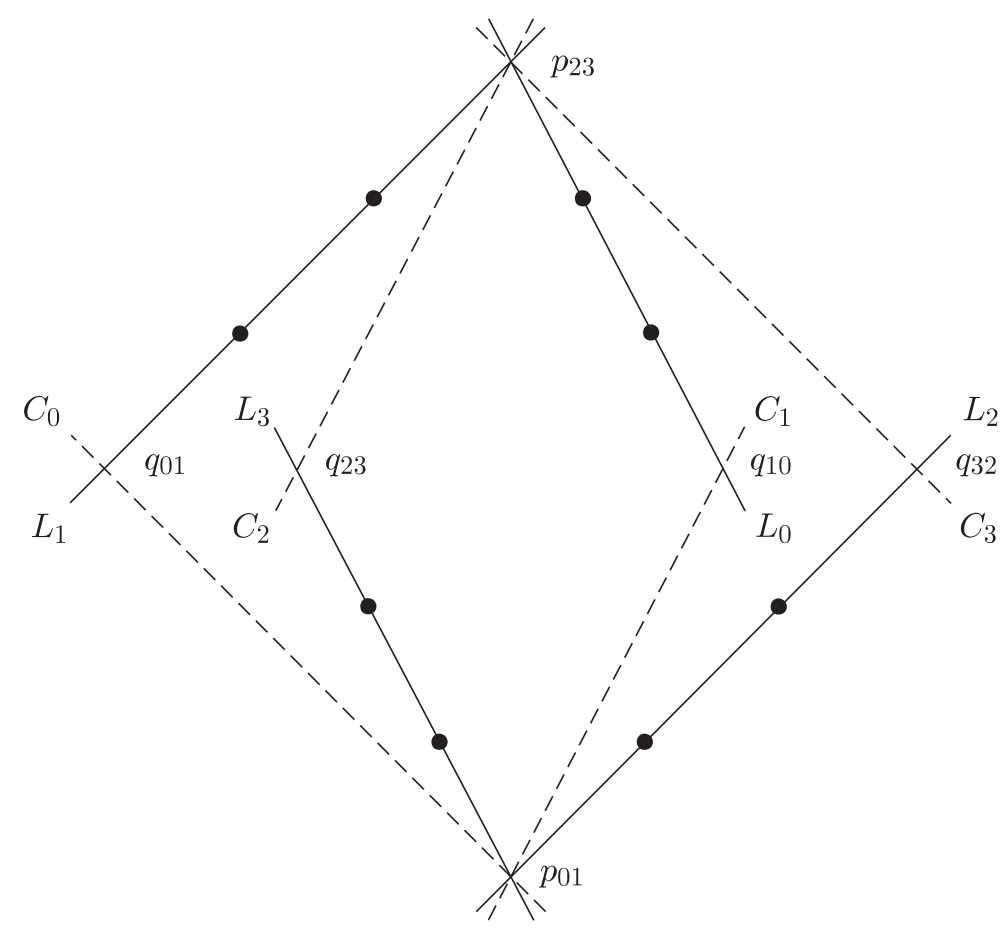

Fig. 3 
R e m a rk 9 . We already know the coordinates of the points $p_{01}, p_{23}$. The other four points form an orbit under the group operation. In the sequel we need the projective coordinates of one of them:

$$
q_{23}=(0: 0: \alpha: 0: 0: \beta),
$$

where $(\alpha: \beta) \in \mathbb{P}^{1}$ depends a priori on the elliptic curves $F_{1}, F_{2}$. In fact, we will show later that $(\alpha: \beta)$ only depends on the curve $F_{1}$, namely $\alpha^{2} / \beta^{2}$ turns out to be its cross-ratio.

5. The equations. Let us use the notation $u_{3}=s_{1}^{2} s_{2}^{2}, u_{4}=s_{0}^{2} s_{3}^{2}$ introduced in [S1] and begin with the following

LEMMA 10. There are complex numbers $\mu_{1}$ and $\mu_{2}$ such that $v:=u_{3}-u_{4}$ $=\mu_{1}\left(w_{5}-w_{6}\right)+\mu_{2} w_{4}$.

$\operatorname{Pro}$ f. It is enough to notice that $v$ is $\sigma$-antiinvariant and $w_{5}-w_{6}, w_{4}$ are a basis for the -1-eigenspace of $\sigma$ according to Lemma 2 and Proposition 4. In fact, we will show later that $\mu_{2}=0$.

There are two obvious quadrics containing the image surface $X$ :

- $Q_{1}=\left\{w_{1} w_{2}-w_{5} w_{6}=0\right\}$,

- $Q_{2}=\left\{w_{1} w_{2}-u_{3} u_{4}=0\right\}=\left\{4 w_{1} w_{2}-w_{3}^{2}+\left(\mu_{1}\left(w_{5}-w_{6}\right)+\mu_{2} w_{4}\right)^{2}=0\right\}$.

To find the next equation let us consider the divisors of the two sections shown in Figure 4.

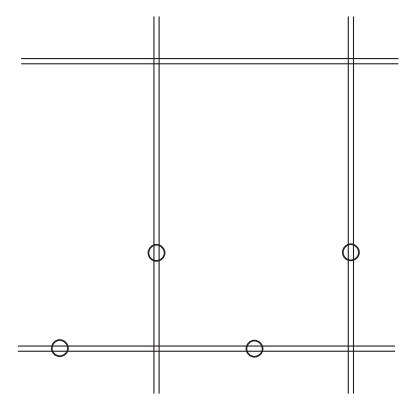

$y_{1}=s_{0}^{2} s_{14}^{2}=s_{1}^{2} s_{12}^{2}$

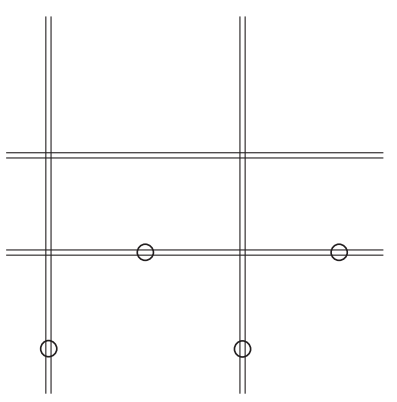

$y_{2}=s_{2}^{2} s_{11}^{2}=s_{3}^{2} s_{9}^{2}$

Fig. 4

These sections are in $L$. Furthermore, they are $\sigma$-invariant and $\tau$ exchanges them without changing the sign. Hence there are complex numbers $\lambda_{1}, \ldots, \lambda_{6}$ such that

$$
y_{1}=\sum \lambda_{i} w_{i} .
$$


Restricting this equality to $F_{1}$ we get immediately $\lambda_{2}=0$ and from $\sigma$ invariance we have $\lambda_{4}=0, \lambda_{5}=\lambda_{6}$. Let $\lambda_{1}=a, \lambda_{3}=b, \lambda_{5}=c$. Then

$$
\begin{aligned}
& y_{1}=a w_{1}+b w_{3}+c\left(w_{5}+w_{6}\right), \\
& y_{2}=-a w_{2}-b w_{3}+c\left(w_{5}+w_{6}\right) .
\end{aligned}
$$

Since $\operatorname{div}\left(w_{4}^{2}\right)=\operatorname{div}\left(y_{1}^{2}\right)+\operatorname{div}\left(y_{2}^{2}\right)$ we are in a position to write down our third equation:

$$
Q_{3}=\left\{w_{4}^{2}+\left(a w_{1}+b w_{3}+c\left(w_{5}+w_{6}\right)\right)\left(a w_{2}+b w_{3}-c\left(w_{5}+w_{6}\right)\right)=0\right\} .
$$

Remark 11. Notice that in the above equation the parameters $a, b, c$ can be considered only up to a multiplicative constant, hence as a point $(a: b: c) \in \mathbb{P}^{2}$. The reason is that $w_{4}$ as a section is fixed only up to a constant.

In the rest of this section we state relations between the parameters $\alpha$, $\beta, \mu_{1}, \mu_{2}, a, b, c$ appearing in our equations. As expected there will be only three (homogeneous) left, depending on the moduli of elliptic curves defining $A$.

To get the relations between the parameters we have to use the information coded in the singular locus of $X$.

Let $x=s p_{01}+t q_{23},(s: t) \in \mathbb{P}^{1}$, be a point on $L_{3}$ and let

$$
W=\left(\begin{array}{c}
\frac{d Q_{1}}{d\left(w_{1}, \ldots, w_{6}\right)}(x) \\
\frac{d Q_{2}}{d\left(w_{1}, \ldots, w_{6}\right)}(x) \\
\frac{d Q_{3}}{d\left(w_{1}, \ldots, w_{6}\right)}(x)
\end{array}\right) .
$$

We can compute explicitly $W$ to be

$$
\left(\begin{array}{cccccc}
s & 0 & 0 & 0 & -\beta t & 0 \\
2 s & 0 & -\alpha t & -t \beta \mu_{1} \mu_{2} & -\mu_{1}^{2} \beta t & \mu_{1}^{2} \beta t \\
a(a s+b t \alpha-c t \beta) & a b t \alpha+a c t \beta & a b s+2 b^{2} t \alpha & 0 & a c s-2 c^{2} t \beta & a c s-2 c^{2} t \beta
\end{array}\right) .
$$

This matrix carries much information about the Kummer surface $X$. Let $W_{i j k}$ denote the minor of $W$ consisting of the $i$ th, $j$ th and $k$ th column of $W$. At the singular points on the line $L_{3}$ all these determinants must vanish. Thus we get a system of degree 3 equations in $s$ and $t$. The crucial observation is that there are three distinct singularities on this line. Hence the obtained equations must be either trivial or have exactly 3 different zeroes. Evaluating this information we get the following conditions:

- $a, b, c, \alpha, \beta, \mu_{1} \neq 0, b^{2} \neq c^{2}, 4\left(b^{2}-c^{2}\right) \neq a^{2}$,

- $\mu_{2}=0$,

- $\alpha b+\beta c=0, \mu_{1}^{2} \beta b+\alpha c=0, \mu_{1}^{2} b^{2}-c^{2}=0$.

Calculations leading to the above conditions are tedious and therefore omitted here. In what follows we set $\mu_{1}^{2}=c^{2} / b^{2}$ and $\beta=-b, \alpha=c$. 
In the next section and in [S2] we need the equation of singularities on $L_{3}$, which we get from $\operatorname{det} W_{156}=0$ :

$$
2 a b^{2} c^{2} t^{3}-b c\left(4 c^{2}-4 b^{2}-a^{2}\right) s t^{2}+2 a\left(b^{2}-c^{2}\right) s^{2} t=0 .
$$

We conclude this section with the following

Proposition 12. The reducible intermediate Kummer surface $X$ in $\mathbb{P}^{5}$ is a complete intersection of a net of quadrics spanned by

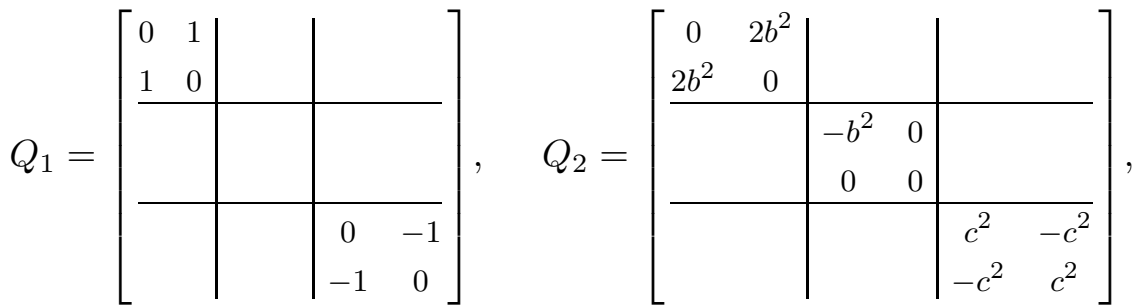

$$
\begin{aligned}
& Q_{3}=\left[\begin{array}{cc|cc|cc}
0 & a^{2} & a b & 0 & -a c & -a c \\
a^{2} & 0 & a b & 0 & a c & a c \\
\hline a b & a b & 2 b^{2} & 0 & 0 & 0 \\
0 & 0 & 0 & 2 & 0 & 0 \\
\hline-a c & a c & 0 & 0 & -2 c^{2} & -2 c^{2} \\
-a c & a c & 0 & 0 & -2 c^{2} & -2 c^{2}
\end{array}\right]
\end{aligned}
$$

where $(a: b: c) \in \mathbb{P}^{2}$ depends on elliptic curves defining the abelian surface $A$ and satisfies $b^{2} \neq c^{2}, 4\left(b^{2}-c^{2}\right) \neq a^{2}, a, b, c \neq 0$.

6. Parameters vs cross-ratios. In this section we show how the parameters $a, b, c$ depend on the moduli of the elliptic curves $F_{1}, F_{2}$ defining $A$. We begin with the following

Lemma 13. $C:=\varphi\left(t_{e_{8}}^{*} F_{1}\right)$ is a conic.

Proof. $\operatorname{deg} C=C \cdot \mathcal{M}^{+}=\frac{1}{2}\left(4 B l^{*}\left(F_{1}+F_{2}\right)-2\left(E_{0}+\ldots+E_{3}\right)\right) \cdot t_{e_{8}}^{*} F_{1}=2$.

Our aim is to parametrize the conic $C$. Let $E$ denote the plane spanned by the conic. Then we have

Lemma 14. The equations of $E$ are

$$
\begin{aligned}
& w_{4}=0, \\
& w_{2}=4 \frac{b^{2}-c^{2}}{a^{2}} w_{1}+2 \frac{c}{a} w_{5}+2 \frac{c}{a} w_{6}, \\
& w_{3}=4 \frac{c^{2}-b^{2}}{a b} w_{1}-\frac{c}{b} w_{5}-\frac{c}{b} w_{6} .
\end{aligned}
$$


Pr o of. From the equation (1) and the action of $G$ we get easily

$$
\begin{aligned}
\varphi\left(e_{8}\right) & =\left(-a b c: 0: 2 c\left(b^{2}-c^{2}\right): 0: 0: 2 b\left(b^{2}-c^{2}\right)\right), \\
\varphi\left(e_{9}\right) & =(0: 2 b c:-a c: 0: 0: a b), \\
\varphi\left(e_{10}\right) & =\left(-a b c: 0: 2 c\left(b^{2}-c^{2}\right): 0: 2 b\left(b^{2}-c^{2}\right): 0\right), \\
\varphi\left(e_{11}\right) & =(0: 2 b c:-a c: 0: a b: 0) .
\end{aligned}
$$

Now to verify our assertion one has to solve a system of linear equations.

Using the above lemma we can parametrize the plane $E$ in the following way:

$$
\begin{gathered}
w_{1}=a^{2} b x, \quad w_{2}=4 b\left(b^{2}-c^{2}\right) x+2 a b c y+2 a b c z \\
w_{3}=4 a\left(c^{2}-b^{2}\right) x-a^{2} c y-a^{2} c z, \quad w_{4}=0, \quad w_{5}=a^{2} b y, \quad w_{6}=a^{2} b z .
\end{gathered}
$$

In the coordinates $(x: y: z)$ the conic $C$ is given by the equation

$$
4\left(b^{2}-c^{2}\right) x^{2}+2 a c x y+2 a c x z-a^{2} y z=0
$$

and the four points determining the cross-ratio for $F_{1}$ are

$$
(0: 0: 1), \quad(0: 1: 0), \quad\left(-a c: 0: 2\left(b^{2}-c^{2}\right)\right), \quad\left(-a c: 2\left(b^{2}-c^{2}\right): 0\right) \text {. }
$$

One verifies easily that the following is a parametrization of the conic $C$

$$
x=a s t, \quad y=2 b s^{2}-2 c s t, \quad z=2 c s t+2 b t^{2},
$$

and the four points in the $(s: t)$-coordinates are $(0: 1),(1: 0),(c:-b)$, $(b:-c)$.

Now we are in a position to state the following

Proposition 15. The cross-ratios $r_{1}, r_{2}$ of the elliptic curves $F_{1}, F_{2}$ are given by $r_{1}=c^{2} / b^{2}$ and $r_{2}=4\left(b^{2}-c^{2}\right) / a^{2}$.

Pro of. For $F_{1}$ there is nothing to do because of the above considerations. For $F_{2}$ we first observe that the mapping $\varphi \mid F_{2}: F_{2} \rightarrow L_{3}$ is a $2: 1$ covering branched over 4 points. To know $F_{2}$ it is enough to compute the cross-ratio of the branch points. Two of them are $t_{1}=q_{23}=(0: 1)$ and $t_{2}=p_{01}=(1: 0)$ written in the $(s: t)$ coordinates and the two others are the $A_{1}$ singularities $t_{3}=\varphi\left(e_{9}\right), t_{4}=\varphi\left(e_{13}\right)$. Their coordinates can be easily computed from equation (1). Thus the cross-ratio is

$$
r_{2}=\frac{b c\left(4 c^{2}-4 b^{2}-a^{2}\right)+\Delta^{1 / 2}}{b c\left(4 c^{2}-4 b^{2}-a^{2}\right)-\Delta^{1 / 2}}
$$

where $\Delta=\left(b c\left(4\left(c^{2}-b^{2}\right)+a^{2}\right)\right)^{2}$. This proves the assertion for $r_{2}$.

Acknowledgements. I would like to thank Prof. W. Barth for turning my attention to the subject presented here and for patient remarks on various versions. I am also grateful to him and Th. Bauer for helpful comments on the manuscript. 


\section{References}

[AvM1] M. Adler and P. van Moerbeke, Geodesic flow on SO(4) and intersection of quadrics, Proc. Nat. Acad. Sci. U.S.A. 81 (1984), 4613-4616.

[AvM2] -, - The intersection of four quadrics in $\mathbb{P}^{6}$, abelian surfaces and their moduli, Math. Ann. 279 (1987), 25-85.

[B] W. Barth, Affine parts of abelian surfaces as complete intersections of four quadrics, ibid. 278 (1987), 117-131.

[BPV] W. Barth, C. Peters and A. van de Ven, Compact Complex Surfaces, Springer, Berlin, 1984.

[Ba] Th. Bauer, Projective images of Kummer surfaces, Math. Ann. 299 (1994), $155-170$

[LB] H. Lange and Ch. Birkenhake, Complex Abelian Varieties, Grundlehren Math. Wiss. 302, Springer, New York, 1992.

[M] D. Mumford, On the equations defining abelian varieties. I, Invent. Math. 1 (1966), 287-354.

[S1] T. Szemberg, $A 4_{3}$ configuration of lines and conics in $\mathbb{P}^{5}$, Ann. Polon. Math. 60 (1995), 145-158.

[S2] - Intersections of quadrics in $\mathbb{P}^{5}$, Kummer surfaces and their moduli, thesis, Erlangen, 1994.

[S-D] B. Saint-Donat, Projective models of K3 surfaces, Amer. J. Math. 96 (1974), 602-639.

INSTITUTE OF MATHEMATICS

JAGIELLONIAN UNIVERSITY

REYMONTA 4

30-059 KRAKÓW, POLAND 